Vietnam Journal of Mechanics, NCST of Vietnam Vol. 25, 2003, No 2 (77-84)

\title{
ON A VARIANT OF THE ASYMPTOTIC PROCEDURE (FOR WEAKLY NONLINEAR AUTONOMOUS SYSTEMS)
}

\author{
NGUYEN VAN Dinh \\ Institute of Mechanics
}

As well-known, usually, in the asymptotic (Krylov-Bogoliubov-Mitropolski) method, the full amplitude (a) of the first harmonic is used as variable in asymptotic expansions [1]. In the first approximation, the equations of stationary oscillations are rather simple, however, in higher approximation, these equations often become very complicated, especially when initial conditions are imposed.

In this article, a variant of the asymptotic procedure is presented and applied to determine stationary oscillations in weakly nonlinear autonomous system with given initial conditions. Instead of the full amplitude, the approximate amplitude of order $\varepsilon^{0}$ is used and by this, stationary oscillations can easily and successively be determined in each step of approximation, although various types of initial conditions may be imposed. It is interesting to note that the results obtained are identical with those given by the Poincaré method [2].

\section{Systems under consideration - The usual asymptotic procedure}

Consider weakly nonlinear autonomous oscillating systems described by following differential equations:

$$
\begin{aligned}
& \ddot{x}+x=\varepsilon f(x), \\
& \ddot{x}+x=\varepsilon f(x, \dot{x}),
\end{aligned}
$$

where $x$ is oscillatory variable; overdots denote differentiation with respect to time $t$; 1 is own frequency; $f(x)$ and $f(x, \dot{x})$-for simplicity-are polynomials of their variables; $\varepsilon$ is a small formal parameter. The equation (1.1) represents weakly nonlinear conservative systems, the equation (1.2) represents weakly nonlinear self-excited systems. The problem of interest is to determine stationary oscillations (free or self-excited oscillations) satisfying initial conditions:

$$
\begin{array}{lll}
\text { for }(1.1): & x(0)=x_{0}, & \dot{x}(0)=0, \\
\text { for }(1.2): & \dot{x}(0)=0 .
\end{array}
$$

For the sake of comparison, the usual procedure of the asymptotic method is briefly recalled: 
First, following asymptotic expansions are used

$$
\begin{aligned}
x & =a \cos \psi+\varepsilon u_{1}(a, \psi)+\varepsilon^{2} u_{2}(a, \psi)+\ldots, \\
\dot{a} & =\varepsilon A_{1}(a)+\varepsilon^{2} A_{2}(a)+\ldots \\
\dot{\psi} & =1+\varepsilon B_{1}(a)+\varepsilon^{2} B_{2}(a)+\ldots,
\end{aligned}
$$

where $a$ is the full amplitude of the first harmonic; $\psi$ is phase angle; $A_{i}, B_{i}$ ( $i=$ $1,2, \ldots)$ are functions of $a$; and $u_{i}(i=1,2, \ldots)$ are functions of $a$ and $\psi$, periodic with respect to $\psi$ with period $2 \pi$.

Since $a$ is the full amplitude of the first harmonic, $u_{i}(i=1,2, \ldots)$ do not contain any first harmonic.

Then, substituting (1.5) into (1.1) or (1.2), using (1.6), (1.7), equating the terms of like powers of $\varepsilon$ yield

$$
\begin{aligned}
& -2 A_{1} \sin \psi-2 a B_{1} \cos \psi+\frac{\partial^{2} u_{1}}{\partial \psi^{2}}+u_{1}=f^{(1)}(a, \psi) \\
& -2 A_{2} \sin \psi-2 a B_{2} \cos \psi+\frac{\partial^{2} u_{2}}{\partial \psi^{2}}+u_{2}=f^{(2)}(a, \psi)=2 A_{1} B_{1} \sin \psi+a B_{1} \frac{\partial B_{1}}{\partial a} \sin \psi \\
& -A_{1} \frac{\partial A_{1}}{\partial a} \cos \psi+a B_{1}^{2} \cos \psi-2 A_{1} \frac{\partial^{2} u_{1}}{\partial \psi \partial a}-2 B_{1} \frac{\partial^{2} u_{1}}{\partial \psi^{2}}+\tilde{f}(a, \psi)
\end{aligned}
$$

where

$$
\begin{aligned}
\text { for }(1.1): f^{(1)}(a, \psi)= & f(a \cos \psi), \quad \tilde{f}(a, \psi)=u_{1} \cdot f_{x}(a \cos \psi) \\
\text { for }(1.2): f^{(1)}(q, \psi)= & f(a \cos \psi,-a \sin \psi) \\
\tilde{f}(a, \psi)= & u_{1}(a, \psi) \cdot f_{x}(a \cos \psi,-a \sin \psi) \\
& +\left(A_{1} \cos \psi-a B_{1} \sin \psi+\frac{\partial u_{1}}{\partial \psi}\right) f_{\dot{x}}(a \cos \psi,-a \sin \psi)
\end{aligned}
$$

$f_{x}, f_{\dot{x}}$ are partial derivatives of the function $f$ with respect to $x, \dot{x}$.

Finally, expanding $f^{(1)}(a, \psi), f^{(2)}(a, \psi)$ in Fourier series, equating the terms of like harmonics yield

$$
\begin{aligned}
& f^{(i)}(a, \psi)=f_{0}^{(i)}(a)+\sum_{n=1}^{N_{i}}\left[S_{n}^{(i)}(a) \sin n \psi+C_{n}^{(i)}(a) \cos n \psi\right] ; N_{i}>0, \text { integer } \\
& A_{i}(a)=-\frac{1}{2} S_{1}^{(i)}(a), \quad B_{i}(a)=-\frac{1}{2 a} C_{1}^{(i)}(a) \\
& \frac{\partial^{2} u_{i}}{\partial \psi^{2}}+u_{i}=f_{0}^{(i)}(a)+\sum_{n=2}^{N_{i}}\left[S_{n}^{(i)}(a) \sin n \psi+C_{n}^{(i)}(a) \cos n \psi\right] \\
& u_{i}(a, \psi)=f_{0}^{(i)}(a)-\sum_{n=2}^{N_{i}} \frac{1}{n^{2}-1}\left[S_{n}^{(i)}(a) \sin n \psi+C_{n}^{(i)}(a) \cos n \psi\right]
\end{aligned}
$$


where $S_{n}^{(i)}(a) \equiv 0$ for the cases (1.1).

Let us determine stationary oscillation in the second approximation.

For (1.1): Since $A_{1}(a) \equiv 0, A_{2}(a) \equiv 0$, without initial condition, the amplitude $a$ and the initial phase $\theta$ are arbitrary and we have

$$
\begin{aligned}
& x=a \cos \psi+\varepsilon\left\{f_{0}^{(1)}(a)-\sum_{n=2}^{N_{1}} \frac{1}{n^{2}-1} C_{n}^{(1)}(a) \cos n \psi\right\} \\
& \psi=\left\{1+\varepsilon B_{1}(a)+\varepsilon^{2} B_{2}(a)\right\} t+\theta .
\end{aligned}
$$

If the initial condition (1.3) are imposed, $a$ and $\theta$ are determined by the equations:

$$
\begin{aligned}
& x(0)=a \cos \theta+\varepsilon\left\{f_{0}^{(1)}(a)-\sum_{n=2}^{N_{1}} \frac{1}{n^{2}-1} C_{n}^{(1)}(a) \cos n \theta\right\}=x_{0}, \\
& \frac{\partial x(a, \theta)}{\partial \psi}=-a \sin \theta+\varepsilon \sum_{n=2}^{N_{1}} \frac{n}{n^{2}-1} C_{n}^{(1)}(a) \sin n \theta=0 .
\end{aligned}
$$

Stationary oscillation is stable with respect to $a$ (not asymptotically)

For (1.2): The amplitude $a$ is determined by the equation

$$
\varepsilon A_{1}(a)+\varepsilon^{2} A_{2}(a)=0,
$$

and, with regard that

$$
x=a \cos \psi+\varepsilon\left\{f_{0}^{(1)}(a)-\sum_{n=2}^{N_{1}} \frac{1}{n^{2}-1}\left[S_{n}^{1}(a) \sin n \psi+C_{n}^{1}(a) \cos n \psi\right]\right\},
$$

the initial phase $\theta$ is determined by the equation

$$
\frac{\partial x(a, \theta)}{\partial \psi}=-a \sin \theta-\sum_{n=2}^{N_{1}} \frac{n}{n^{2}-1}\left[S_{n}^{(1)}(a) \cos n \theta-C_{n}^{(1)}(a) \sin n \theta\right]=0
$$

The condition for asymptotic stability is

$$
\varepsilon \frac{\partial A_{1}}{\partial a}+\varepsilon^{2} \frac{\partial A_{2}}{\partial a}<0
$$

\section{Stationary oscillation from a variant of the asymptotic procedure}

In this section, the problem of interest is treated by a variant of the asymptotic procedure.

First, $a$ is considered now as the approximate (not full) amplitude of order $\varepsilon^{O}$ of the first harmonic. The asymptotic expansions of $x, \dot{x}, \dot{\psi}$ retain their forms (1.5), (1.6), (1.7) but the functions $u_{i}(i=1,2, \ldots)$ may contain the first harmonics $a_{i} \cos \psi+b_{i} \sin \psi$ of order $\varepsilon^{i}$ where $a_{i}, b_{i}$ are constants to be chosen. The presence of $a_{i}, b_{i}$ modifies all the calculations in the second and higher approximations; however, as "compansion", $a$ suitable choice of $a_{i}, b_{i}$ allows us to consider the initial phase as zero, that is $\psi(0)=0$. 
Then, stationary oscillation is determined - not at the end but - successively in each step of approximation; this can be done by using two following requirements:

1 - The amplitude $a$ (of stationary oscillation) is constant i.e. $\dot{a}=0$ in each step of approximation; this means that, for every $i$, we have

$$
A_{i}\left(a_{*}\right)=0 \quad(i=1,2, \ldots) .
$$

2 - Also in each step of approximation, the initial conditions must by satisfied.

For the initial condition $x(0)=x_{0}$, the amplitude of stationary oscillation should be taken

$$
a=a_{*}=x_{0}
$$

and the functions $u_{i}$ should be vanished at the initial moment, that is

$$
u_{i}\left(a_{*}, \psi(0)\right)=u_{i}\left(a_{*}, 0\right)=0 \text {. }
$$

For the initial condition $\dot{x}(0)=0$, the partial derivative of $u_{i}$ with respect to $\psi$ at initial moment should be vanished, that is

$$
\frac{\partial u_{i}\left(a_{*}, 0\right)}{\partial \psi}=0
$$

In detail, for (1.1), in the first approximation, $x=a \cos \psi$ and since $A_{1}(a) \equiv 0$, we have $a=a_{*}=x_{0}$, the initial conditions $x(0)=x_{0}, \dot{x}(0)=0$ are satisfied. The expression of $u_{1}(a, \psi)$ is of the form:

$$
u_{1}(a, \psi)=f_{0}^{(1)}(a)-\sum_{n=2}^{N_{1}} \frac{1}{n^{2}-1} C_{n}^{(1)}(a) \cos n \psi+a_{1} \cos \psi+b_{1} \sin \psi
$$

Again, to satisfy the initial conditions (1.3), $a_{1}$ and $b_{1}$ should be taken

$$
a_{1}=a_{1 *}=-f_{0}^{(1)}\left(a_{*}\right)+\sum_{n=2}^{N_{1}} \frac{1}{n^{2}-1} C_{n}^{(1)}\left(a_{*}\right) ; \quad b_{1}=b_{1 *}=0 .
$$

Because of the presence of $a_{1 *}$ in $u_{1}(a, \psi)$, the expansion of $f^{(2)}(a, \psi)$ is modified

$$
f^{(2)}(a, \psi)=\bar{f}_{0}^{(2)}(a)+\sum_{n=1}^{N_{2}} \bar{C}_{n}^{(2)}(a) \cos n \psi
$$

where $\bar{f}_{0}^{(2)}(a), \bar{C}_{n}^{(2)}(a)$ differ from $f_{0}^{(2)}(a), C_{n}^{(2)}(a)$.

In the second approximation

$$
\begin{aligned}
& A_{2}(a) \equiv 0, \quad B_{2}(a)=-\frac{1}{2 a} \bar{C}_{1}^{(2)}(a) \\
& u_{2}(a, \psi)=\bar{f}_{0}^{(2)}(a)-\sum_{n=2}^{N_{2}} \frac{1}{n^{2}-1} \bar{C}_{n}^{(2)}(a) \cos n \psi+a_{2} \cos \psi+b_{2} \sin \psi
\end{aligned}
$$


where $\quad a_{2}=a_{2 *}=-\bar{f}_{0}^{(2)}\left(a_{*}\right)+\sum_{n=2}^{N_{2}} \frac{1}{n^{2}-1} \bar{C}_{n}^{(2)}\left(a_{*}\right), \quad b_{2}=b_{2 *}=0$.

For (1.2): In the first approximation, the amplitude $a_{*}$ of stationary oscillation is determined by the equation

$$
\varepsilon A_{1}(a)=0 \text { i.e. } S_{1}^{(1)}(a)=0 .
$$

The expansion of $u_{1}(a, \psi)$ is:

$u_{1}(a, \psi)=f_{0}^{(1)}(a)-\sum_{n=2}^{N_{1}} \frac{1}{n^{2}-1}\left[S_{n}^{(1)}(a) \sin n \psi+C_{n}^{(1)}(a) \cos n \psi\right]+a_{1} \cos \psi+b_{1} \sin \psi$.

To satisfy the initial condition (1.4), $b_{1}$ should be chosen such that

$$
\frac{\partial u_{1}\left(a_{*}, 0\right)}{\partial \psi}=0 \text { i.e. } \quad b_{1}=b_{1 *}=\sum_{n=2}^{N_{1}} \frac{n}{n^{2}-1} S_{n}^{(1)}\left(a_{*}\right) .
$$

As to $a_{1}$, it can only be determined in the second approximation. Indeed, the expansion of $f^{(2)}(a, \psi)$ can be written as

$$
\begin{aligned}
f^{(2)}(a, \psi)= & {\left[\bar{f}_{0}^{(2)}(a)+a_{1} f_{0}(a)\right] } \\
& +\sum_{n=1}^{N_{2}}\left\{\left[\bar{S}_{n}^{(2)}(a)+a_{1} S_{n}(a)\right] \sin n \psi+\left[\bar{C}_{n}^{(2)}(a)+a_{1} C_{n}(a)\right] \cos n \psi\right\}
\end{aligned}
$$

and $\quad A_{2}(a)=\frac{-1}{2}\left[\bar{S}_{1}^{(2)}(a)+a_{1} S_{1}(a)\right], \quad B_{2}=\frac{-1}{2 a}\left[C_{1}^{(2)}(a)+a_{1} C_{1}(a)\right]$.

Imposing on $A_{2}(a)$ the requirement $A_{2}\left(a_{*}\right)=0$, we obtain

$$
a_{1}=a_{1 *}=-\frac{\bar{S}_{1}^{(2)}\left(a_{*}\right)}{S_{1}\left(a_{*}\right)} \text { on condition that } S_{1}\left(a_{*}\right) \neq 0 .
$$

Thus, in the second approximation, we have

$$
\begin{aligned}
x=a \cos \psi+\varepsilon\left\{f_{0}^{(1)}(a)-\sum_{n=2}^{N_{1}}\left[S_{n}^{(1)}(a) \sin n \psi+C_{n}^{(1)}(a) \cos n \psi\right]\right. \\
\left.\quad+a_{1 *} \cos \psi+b_{1 *} \sin \psi\right\}, \\
\dot{a}=\varepsilon A_{1}(a)+\varepsilon^{2} A_{2}(a)=-\frac{\varepsilon}{2} S_{1}^{(1)}(a)-\frac{\varepsilon^{2}}{2}\left\{\bar{S}_{1}^{(2)}(a)+a_{1 *} S_{1}(a)\right\}, \\
\dot{\psi}=1+\varepsilon B_{1}(a)+\varepsilon^{2} B_{2}(a)=1-\frac{\varepsilon}{2 a} C_{1}^{(1)}(a)-\frac{\varepsilon^{2}}{2 a}\left\{\bar{C}_{1}^{(2)}(a)+a_{1 *} C_{1}(a)\right\} .
\end{aligned}
$$

The amplitude $a_{*}$ of stationary oscillation is given by (2.11) and the phase angle of stationary oscillation is $\psi(t)=\left\{1+\varepsilon B_{1}\left(a_{*}\right)+\varepsilon^{2} B_{2}\left(a_{*}\right)\right\} t$. Note that in (2:17), 
(2.18), (2.19), $a=a(t)$ is the varying amplitude therefore, from (2.18), the condition for asymptotically stability can easily be established

$$
\left(\varepsilon \frac{\partial A_{1}}{\partial a}+\varepsilon^{2} \frac{\partial A_{2}}{\partial a}\right)_{*}<0 .
$$

The expression of $A_{2}$ in (2.20) differs from that corresponding in (1.21). On the other hand, the left hand side of (2.20) is calculated with $a=a_{*}$ determined in the first approximation while the left hand side of (1.21) is calculated with a determined in the second approximation. Thus, (2.20)-being not identical with (1.21) - is more simple and that is just an advantage of the variant of the asymptotic method.

\section{Comparison and example}

The modified procedure already presented can be justified by comparing the results obtained in $\S 2$ with those given by the Poincaré method [2]. Indeed, for the case (1.2), introducing the new time $\tau$

$$
\tau=\omega t
$$

the system (1.1), (1.2) are rewritten as

$$
\begin{aligned}
& \omega^{2} x^{\prime \prime}+x=\varepsilon f\left(x, \omega x^{\prime}\right), \\
& x^{\prime}(0)=0,
\end{aligned}
$$

where $\omega$ is the unknown frequency; primes denote differentiation with respect to $\tau$.

Then both two unknowns $x$ and $\omega$ are expanded in powers of $\varepsilon$ that is

$$
\begin{aligned}
& x=x_{0}+\varepsilon x_{1}+\varepsilon^{2} x_{2}+\ldots, \\
& \omega=1+\varepsilon \omega_{1}+\varepsilon^{2} \omega_{2}+\ldots
\end{aligned}
$$

As to the initial condition (1.4), it is replaced by

$$
\begin{aligned}
& x_{0}^{\prime}(0)=0, \\
& x_{i}^{\prime}(0)=0, \quad(i=1,2, \ldots) .
\end{aligned}
$$

Substituting (3.4), (3.5) into (3.2), expanding the right hand side in Taylor's series of $\varepsilon$, equating the terms of like powers of $\varepsilon$ in the two sides yield

$$
\begin{aligned}
& x_{0}^{\prime \prime}+x_{0}=0, \\
& x_{1}^{\prime \prime}+x_{1}+2 \omega_{1} x_{0}^{\prime \prime}=f\left(x_{0}, x_{0}^{\prime}\right), \\
& x_{2}^{\prime \prime}+x_{2}+2 \omega_{2} x_{0}^{\prime \prime}=-2 \omega_{1} x_{0}^{\prime \prime}-\omega_{1}^{2} x_{0}^{\prime \prime}+x_{1} f_{x}+\left(\omega_{1} x_{0}^{\prime}+x_{1}^{\prime}\right) f_{x}\left(x_{0}, x_{0}^{\prime}\right), \\
& \ldots \quad \ldots \quad \ldots
\end{aligned}
$$


The general solution of the differential equation (3.8) satisfying the initial condition (3.6) is

$$
x_{0}=a \cos \tau \text {. }
$$

Substituting (3.11) into (3.9), (3.10) gives

$$
\begin{aligned}
x_{1}^{\prime \prime}+x_{1}-2 a \omega_{1} \cos \tau= & f\left(a \cos \tau_{1}-a \sin \tau_{1}\right), \\
x_{2}^{\prime \prime}+x_{2}-2 a \omega_{2} \cos \tau= & 2 a \omega_{1} \cos \tau+a \omega_{1}^{2} \cos \tau+x_{1} f_{x}\left(a \cos \tau_{1}-a \sin \tau_{1}\right) \\
& +\left(-a \omega_{1} \sin \tau+x_{1}^{\prime}\right) f_{\dot{x}}\left(a \cos \tau_{1}-a \sin \tau\right),
\end{aligned}
$$

Except the absence of the terms containing $A_{1}, A_{2}$ as factors, the difference between the equations (3.12), (3.13) and their corresponding ones (1.8), (1.9) is of formal character and consists only in the difference between the notations $\left(\tau=\omega t, \omega_{i}\right.$, $x_{i}$ and $\left.\psi=\omega t, B_{i}, u_{i}\right)$. However, for steady state, $A_{1}\left(a_{*}\right)=A_{2}\left(a_{*}\right)=0$; so, the equations (3.12), (3.13) lead to the same results as those in $\S 2$ i.e. the stationary oscillation obtained in $\S 2$ is identical with that determined by the Poincare method.

As an illustration, consider the system

$$
\ddot{x}+x=\varepsilon h\left(1-x^{2}\right) \dot{x}, \quad h>0 .
$$

In the first approximation we have:

$$
\begin{aligned}
& -2 A_{1} \sin \psi-2 a B_{1} \cos \psi+\frac{\partial^{2} u_{1}}{\partial \psi^{2}}+u_{1}=h\left(1-a^{2} \cos ^{2} \psi\right)(-a \sin \psi) \\
& \quad=-h a\left(1-\frac{a^{2}}{4}\right) \sin \psi+\frac{h a^{3}}{4} \sin 3 \psi, \\
& A_{1}(a)=\frac{1}{2} h a\left(1-\frac{a^{2}}{4}\right), \quad B_{1}=0, \quad a_{*}=2, \\
& u_{1}(a, \psi)=-\frac{h a^{3}}{32} \sin 3 \psi+a_{1} \cos \psi+b_{1} \sin \psi, \\
& \frac{\partial u_{1}}{\partial \psi}=-\frac{3 h a^{3}}{32} \cos 3 \psi-a_{1} \sin \psi+b_{1} \cos \psi, \quad b_{1}=\frac{3 h a_{*}^{3}}{32}=\frac{3 h}{4} .
\end{aligned}
$$

In the second approximation, we have:

$$
\begin{aligned}
&- 2 A_{2} \sin \psi-2 a B_{2} \cos \psi+\frac{\partial^{2} u_{2}}{\partial \psi^{2}}+u_{2}=-A_{1} \frac{\partial A_{1}}{\partial a} \cos \psi-2 A_{1} \frac{\partial^{2} u_{1}}{\partial \psi \partial a} \\
&+ u_{1} h a^{2} \sin 2 \psi+\left(A_{1} \cos \psi+\frac{\partial u_{1}}{\partial \psi}\right) h\left(1-a^{2} \cos ^{2} \psi\right) \\
&= h a_{1}\left(\frac{5 a^{2}}{4}-1\right) \sin \psi+\left\{\frac{h^{2} a^{5}}{128}+\frac{3 h^{2}}{4}\left(1-\frac{a^{2}}{4}\right)-A_{1} \frac{\partial A_{1}}{\partial a}+A_{1} h\left(1-\frac{3 a^{2}}{4}\right)\right\} \cos \psi \\
& \quad \text { higher harmonics, }
\end{aligned}
$$




$$
\begin{aligned}
& A_{2}=-\frac{h a_{1}}{2}\left(\frac{5 a^{2}}{4}-1\right) \\
& B_{2}=-\frac{1}{2 a}\left\{\frac{h^{2} a^{5}}{128}+\frac{3 h^{2}}{4}+\frac{3 h^{2}}{4}\left(1-\frac{a^{2}}{4}\right)-A_{1} \frac{\partial A_{1}}{\partial a}+A_{1} h\left(1-\frac{3 a^{2}}{4}\right)\right\} .
\end{aligned}
$$

We choose $a_{1}=0$ so that $A_{2}(a) \equiv 0$. Thus, in the second approximation the expression of $x$ is

and the condition of stability is

$$
x=2 \cos \psi+\varepsilon \frac{3 h}{4} \sin \psi-\varepsilon \frac{h}{4} \sin 3 \psi
$$

$$
\left(\varepsilon \frac{\partial A_{1}}{\partial a}\right)_{*}=-\varepsilon \frac{h a_{*}^{2}}{4}=-\varepsilon h<0 \text { i.e. } h>0 .
$$

\section{Conclusion}

The variant of the asymptotic procedure above presented can be used for studying weakly nonlinear systems with given initial conditions. The determination as well as the stability study of stationary oscillation is rather simple, especially for higher approximation. The results obtained are identical with those given by the Poincaré method.

This publication is completed with the financial support from The Council for Natural Science of Vietnam.

\section{REFERENCES}

1. Bogoliubov N. N., Mitropolskii Yu. A. Asymptotic methods in the theory of nonlinear oscillations, Moscow, 1974.

2. Nayfeh A. N. Pertubation method, Wiley, New York, 1973.

Received December 10, 2002

\section{MỘT BIẾN THỂ CƯA TRÌNH TỰ TIỆM CẬN}

(HỆ ÔTÔNÔM PHI TUYẾN YẾU)

Hệ ôtônôm phi tuyến yếu được khảo sát nhờ một biến thể của trình tự tiệm cận. Thay cho biên độ đầy đủ, biên độ ở xấp xỉ $\varepsilon^{0}$ của ác môníc thứ nhất được sử dụng trong khai triển tiệm cận. Việc xác định cũng như việc khảo sát ổn định của chế độ dừng có phần đơn giản hơn, nhất là ở xấp xỉ bậc cao. Các kết quá thu được trùng với các kết quả tương ứng trong phương pháp Poincaré. 